\title{
correspondence
}

\section{Shining light on intelligence}

SIR,- I agree with Hermann Bondi (16 March, page 204) that "a great deal of heat and little if any light has been generated by discussions of the inheritability or otherwise of intelligence" but I consider that the rest of his letter functions only to cloud the issue, obscuring the little light that exists.

If we attempt to pierce the clouds, it would appear that Bondi's contribution to the discussion takes the following form (1) complex human traits have a smaller genetic component than simple ones; (2) mental attributes are more complex than physical ones; (3) therefore, from (1) and (2), mental attributes are more affected by the environment and less by one's genes than are physical attributes; (4) the environment has a "commanding", part to play in athletic ability; (5) therefore, from (3) and (4), genes can have little part to play in determining human intelligence.

Statement (1) is a little difficult to discuss as Bondi does not define complexity. However, he says some traits "(for example colour of eyes or hair, colour blindness and haemophilia) are easily defined, readily measured, constant throughout life, and it seems to be reasonable to classify them as simple" It is difficult to resist the temptation to question whether hair-colour really satisfies the three criteria laid down but let us accept that these characters are, indeed, simple. Since they are largely under genetic control, they fit in with statement (1). However, two other simple characters that come readily to mind, handedness and tongue-rolling, appear to have very little genetic basis. Statement (1) may be true in general but there are so many exceptions that one cannot use it to predict heritability from "complexity" in any particular case.

Statement (2), at least when referring to physical achievements such as athletic ability, is pure assumption. Though among readers of Nature it may be true that "few would deny" it, this reflects an understandable prejudice rather than the existence of objective data.

Statement (4) rests on a clear misunderstanding of how the interplay of nature and nurture can be studied. The "inheritability" of quantitative characters may be measured as the proportion which the additive genetic variance represents of the phenotype variance. This measureheritability-may not always coincide with what the non-geneticist means by "inheritability" but it is useful in genetic which is, after all, the scientific study of
inheritance.

Bondi's argument, based on the difference in athletic performance between East and West Germans is that the environment must have a commanding part to play in establishing complex physical achievements. If I take two genetically identical populations of an organism, subject them to different environments, and then find that the individuals with the highest scores for some quantitative trait generally come from one of the populations, I can reasonably conclude that the imposed environmental differences have resulted in the observed differences between the populations. But I cannot tell what proportion of the total variance of the trait in question is due to the environmental difference: it may well be small. Indeed, the variance within each population may be entirely genetic. To estimate either the overall heritability or the heritability within populations we need quite different data. This is as true of Bondi's particular case as of the general case.

It would thus appear that statements (1), (2), and (4) are almost totally unfounded. Conclusions (3) and (5) must. therefore, fall. They may eventually turn out to be correct but Bondi's arguments do not help us decide one way or the other.

The problem with measuring the heritability of intelligence is not that it is very small or that the question is complex-after all, we can measure the charge of an electron and discover the amino-acid sequences of proteins. The real problem is that, for perfectly proper reasons, we cannot do the usual experiments. For traits with heritability close to unity, this matters little. For all others, not just intelligence, it matters a lot. Is Bondi advocating that because this problem is consequently difficult, we should not try to solve it?

$$
\text { J. J. D. Greenwood }
$$

University of Dundee, $\dot{U} K$

SIR.-The argument advanced by Sir Hermann Bondi (16 March, page 204) leading to the conclusion that environment "must have a commanding part in establishing complex human physical achievement" is unsound. In the first place comparisons of small numbers of exceptional individuals (such as Olympic athletes) are not particularly informative with respect to the qualities of the population from which they are drawn. More importantly, however, while the athletic achievements of the DDR and some other nations of Eastern Europe are indeed outstanding it seems likely that this owes not a little to the highly selective (not to say elitist) practices which operate in many of these countries. In such an environment individuals displaying creative, intellectual or physical talent tend to be systematically picked out and coached from an early age.

It could thus be argued that the DDR is better than, say, the Federal Republic of Germany at recognising outstanding genotypes and that environmental contribution to the achievements of such individuals is therefore rather small.

Both Sir Hermann's conclusion and the one derived above are overly simplistic. There are other, and scientifically more respectable, viewpoints which take account of the importance of the right environment for the right genotype in achieving an extreme value for any aspect of phenotype. The truth can, of course, only be established by properly planned and executed enquiry from which we should not be deterred by the "incredibly complex" nature of the physical and mental attributes involved. Yours faithfully,

\section{J. R. BEARDMORE}

University College of Swansea, UK

\section{Windscale: preparing MPs for an informed debate}

Sir,-Your editorial (30 March, page 389 ), on the Windscale debate makes a perfectly fair point that an issue of this kind cannot be satisfactorily disposed of by a few hours slanging match across the floor of the House. The subject highlights the need for a powerful and coherent committee system for the House of Commons which at present it lacks.

However, your comments are a good deal less than fair to the work of the Select Committee on Science and Technology, of which I am a member, and which has produced over the past few years a number of important and influential reports on energy problems including a recent one on alternative energy sources commending the development of tidal, wave and solar power. I am inclined to agree that energy is now a subject of sufficient importance to deserve a Select Committee in its own right.

Your editorial does dodge the issue as to who must take the ultimate decision in a matter as important as Windscale. A Select Committee can probe, investigate, weigh evidence and make recommendations, but the House itself must ultimately decide. In my view, the vote before Easter of 186 to 56 probably represented fairly well the current balance of opinion within the House of Commons on the nuclear issue-which does not split on party lines. Nevertheless, I would hope that more work of the kind already carried out by the Select Committee on Science and Technology taken together with the work of other bodies such as the Royal Commission on Environmental Pollution. will eventuallv bring about a substantial shift in this balance.

$$
\text { Yours faithfully, }
$$
Frank HoOLEY, M.P.

House of Commons,

London, UK

\section{Argentinian haematologist released}

News has jut been received that the haematologist Dr Beatriz IparagyuirreWeinstein, who was reported missing in Buenos Aires (30 March, p 396) has now been released by her captors and is safe. 